\title{
Pictorial essay: Role of magnetic resonance imaging in evaluation of brachial plexus pathologies
}

\author{
Malini Lawande, Deepak P Patkar, Sona Pungavkar \\ Department of MRI, Dr. Balabhai Nanavati Hospital, Mumbai, India
}

Correspondence: Dr. Malini A Lawande, 5A, Shimpla Vignaharta Scty, 35, Juhu Versova Link Road, Andheri West, Mumbai - 400053, India. E-mail: malinilawande@gmail.com

\begin{abstract}
Brachial plexopathies, traumatic and nontraumatic, often present with vague symptoms. Clinical examination and electrophysiological studies are useful but may not localize the lesion accurately. Magnetic resonance imaging (MRI) with its multiplanar imaging capability and soft tissue contrast resolution plays an important role in evaluation of the abnormal brachial plexus.
\end{abstract}

Key words: Brachial plexus; MRI; thoracic outlet syndrome

\section{Introduction}

Brachial plexus is the main sensory and motor innervation of the upper extremity. It may be involved in a variety of traumatic and nontraumatic pathologies. MRI is useful both in diagnosis and in localization of these lesions. This makes a significant impact on the management.

Imaging of brachial plexus by MRI is challenging due to the large field of view to be covered with heterogeneous tissue distribution including fat, muscles, and bones.

\section{Normal anatomy}

The brachial plexus is formed by the ventral roots of $\mathrm{C} 5$ to $\mathrm{T} 1$ nerve roots. These unite to form three trunks. The trunks split into three anterior and three posterior divisions. These unite to form the three cords that further divide into five peripheral nerves. The roots and trunks are supraclavicular in location while divisions are retroclavicular and the cords are infraclavicular. ${ }^{[1,2]}$

\begin{tabular}{|l|l|}
\hline \multicolumn{2}{|c|}{ Access this article online } \\
\hline Quick Response Code: & \\
\hline & Website: \\
\hline & www.iji.org \\
& DOI: \\
\hline
\end{tabular}

\section{Imaging technique}

The T1-weighted images delineate the anatomy of nerves, muscles, and vessels as they are outlined by fat, while the T2-weighted images reveal the signal abnormalities within the brachial plexus. Short-tau inversion recovery (STIR) images provide uniform and reliable fat suppression over curved surfaces and large field of view [Figure 1A and B]. The scan protocol followed at our department consists of coronal STIR and T1-weighted images with large field of view (FOV), including both brachial plexi for comparison followed by sagittal T1-and T2-weighted images with small FOV for high spatial resolution. Intravenous Gadolinium is administered in patients with tumors or mass lesions. Gadolinium is not administered in patients with traumatic brachial plexopathy. In patients with traumatic brachial plexus injury, in addition to the previously described protocol, sagittal T2-weighted images are obtained through the

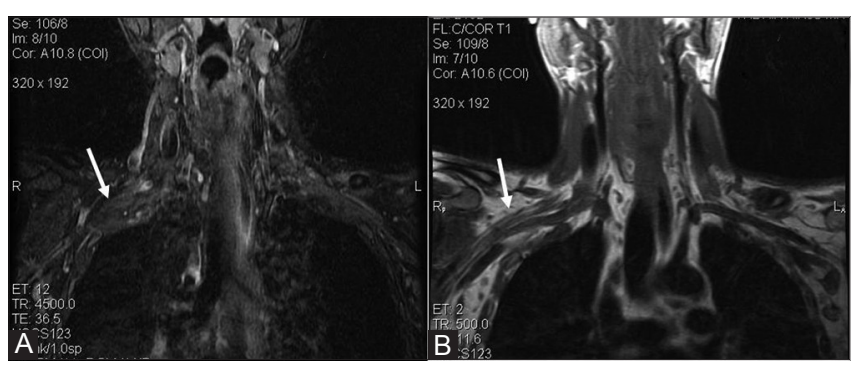

Figure 1 (A, B): Coronal STIR and T1-weighted images demonstrate normal brachial plexus 


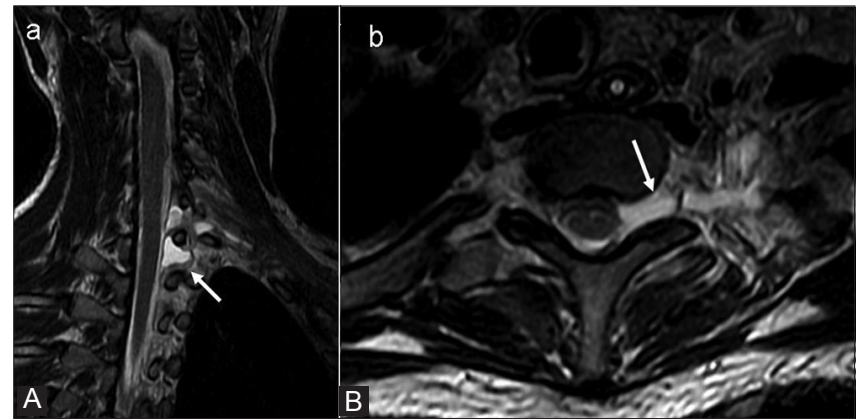

Figure 2 (A, B): Oblique sagittal and axial T2-weighted images reveal pseudomeningoceles at sites of left C7 and C8 nerve root avulsion

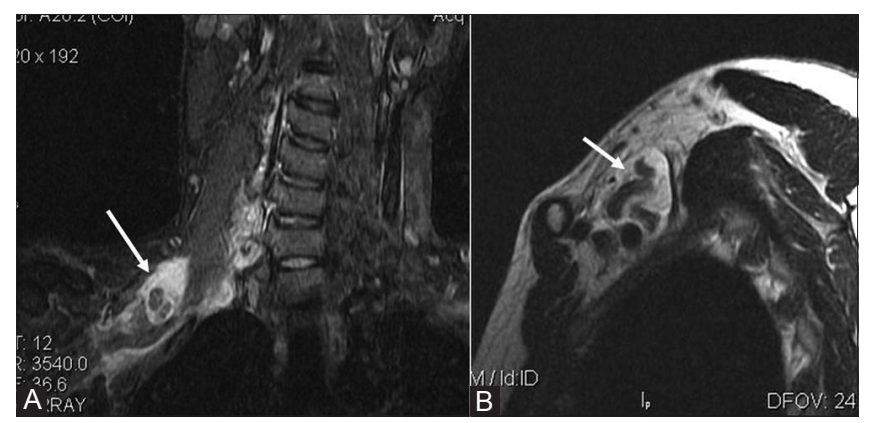

Figure 4 (A, B): Coronal STIR and sagittal T2-weighted images show nerve rupture with fluid collection around the retracted nerves suggestive of postganglionic lesion

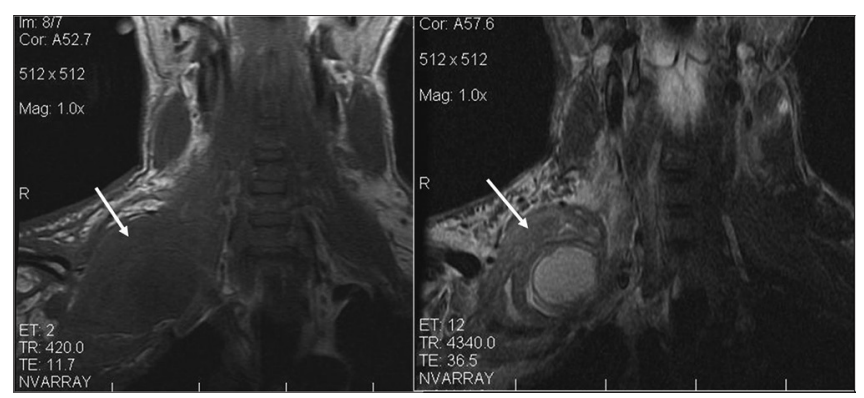

Figure 6: Coronal T1 and STIR images reveal posttraumatic pseudoaneurysm of right subclavian artery causing compression of adjacent right brachial plexus

cervical spine followed by axial T2-weighted images from C4 to T2 levels. In addition, a 3D gradient echo (GRE) sequence with thin slices is obtained to look for the nerve root avulsion.

In patients suspected with thoracic outlet syndrome, in addition to coronal STIR and coronal T1-weighted images, sagittal T1-weighted images are obtained through the symptomatic side extending from midline to the axilla with arm in hyperabducted position. These are compared with similar sagittal T1-weighted images obtained with arm in neutral position by the side of body.

Traumatic injuries to brachial plexus

The common causes of brachial plexus injuries are road traffic accidents and birth palsy. Brachial plexus injuries can be divided into pre- and postganglionic lesions. The

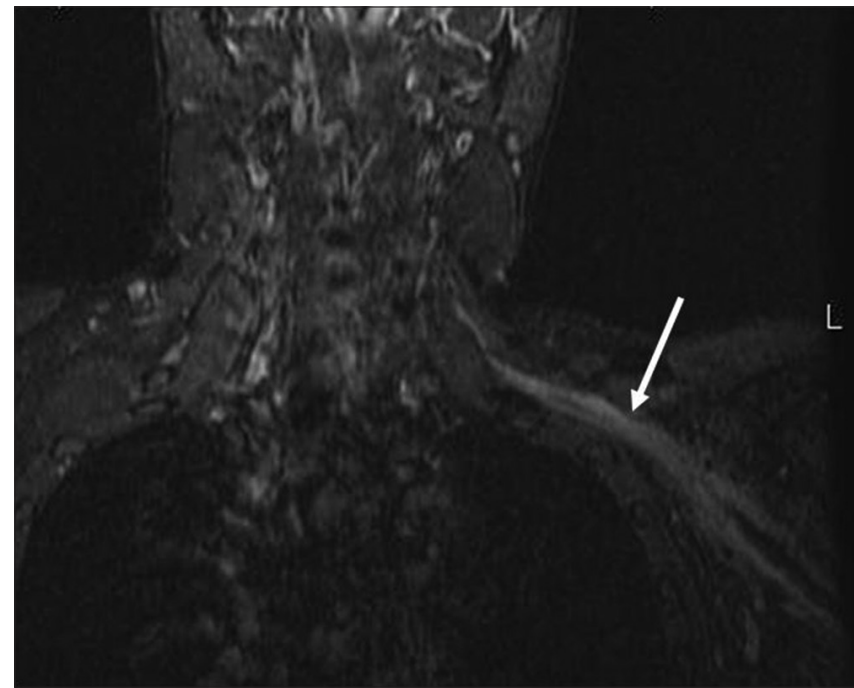

Figure 3: Coronal STIR image reveals swollen hyperintense divisions and cords of left brachial plexus suggestive of postganglionic neuropraxic injury

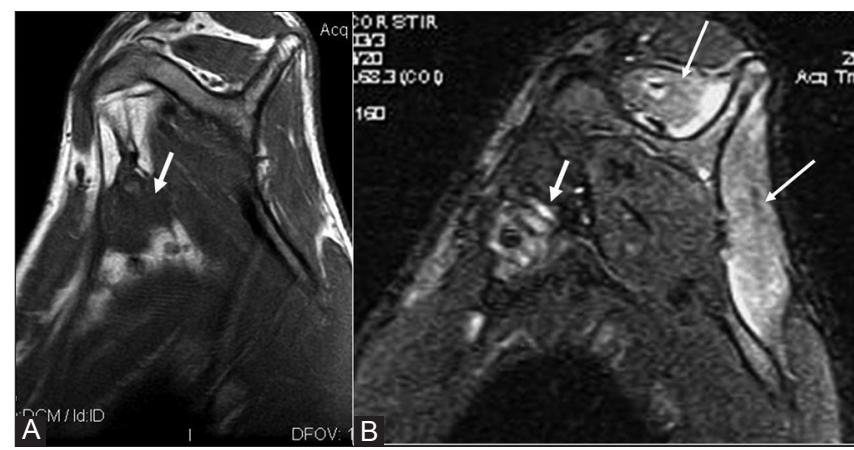

Figure 5 (A, B): Sagittal T1 and STIR images show postganglionic injury with fibrosis (short thick arrows) encasing the cords of left brachial plexus. Note the denervation edema in supra and infraspinatus muscles (thin arrows) seen as ill-defined hyperintense signal on STIR images

preganglionic lesions are avulsion of the nerve roots at their origin while postganglionic lesions may be lesions in continuity or nerve ruptures. The patient may have a combination of both pre- and postganglionic lesions. It is important to differentiate between pre and postganglionic lesions to determine the prognosis and plan further management. Postganglionic lesions in continuity without disruption of nerve fibers have good prognosis and recover spontaneously with conservative management. Postganglionic lesions with disruption of nerve fibers are treated with surgical repair, that is, nerve grafting with good results. Preganglionic lesions are usually treated with nerve transfers to restore function of the denervated muscles. ${ }^{[3,4]}$

Pseudomeningoceles are formed due to extravasation of cerebrospinal fluid through tear of the perineural sheath. These are seen on T2-weighted images as fluid-intensity lesions at the site of nerve root avulsion [Figure 2A and B]. However, presence of a pseudomeningocele is not always seen in nerve root avulsion and vice versa. 


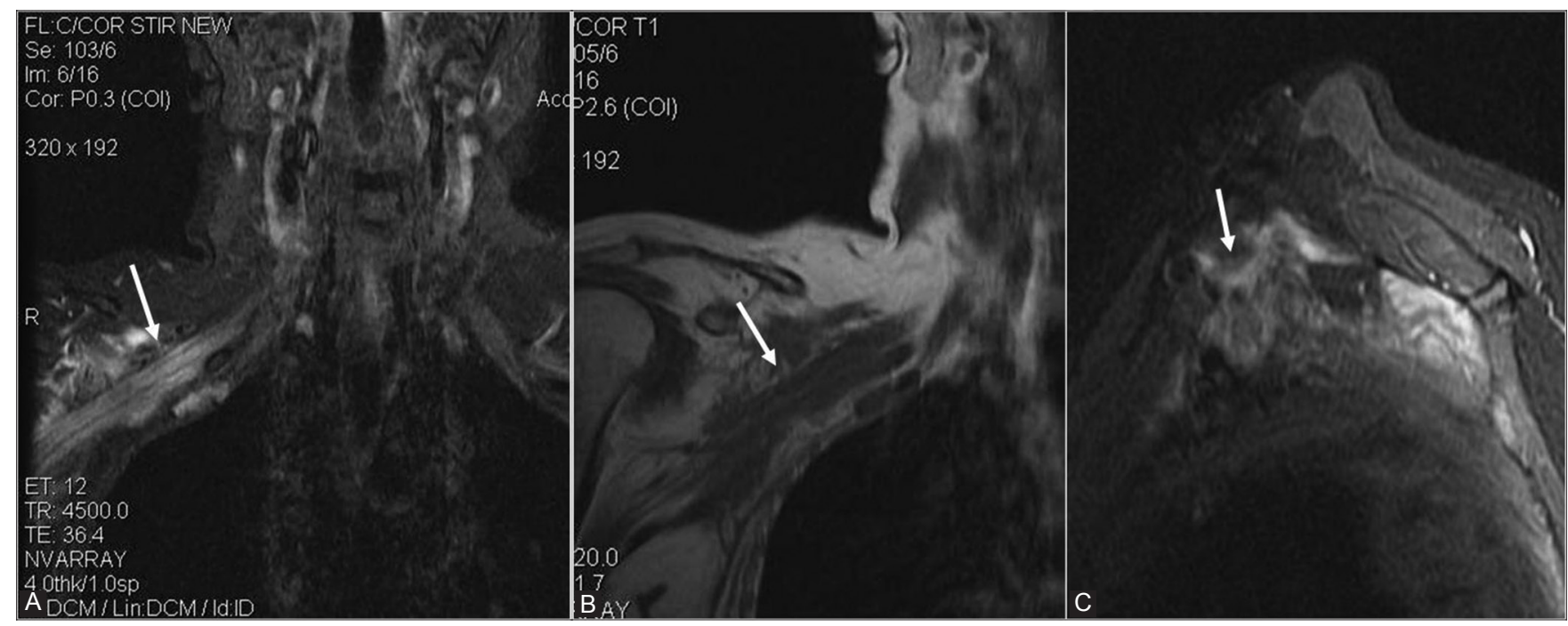

Figure 7 (A-C): Coronal STIR, coronal T1-weighted and postcontrast sagittal fat-saturated T1-weighted images show radiation fibrosis as diffuse thickening of divisions and cords of brachial plexus. They reveal hyperintense signal on STIR image and reveal moderate enhancement. There is no focal mass lesion, thus differentiating it from metastases

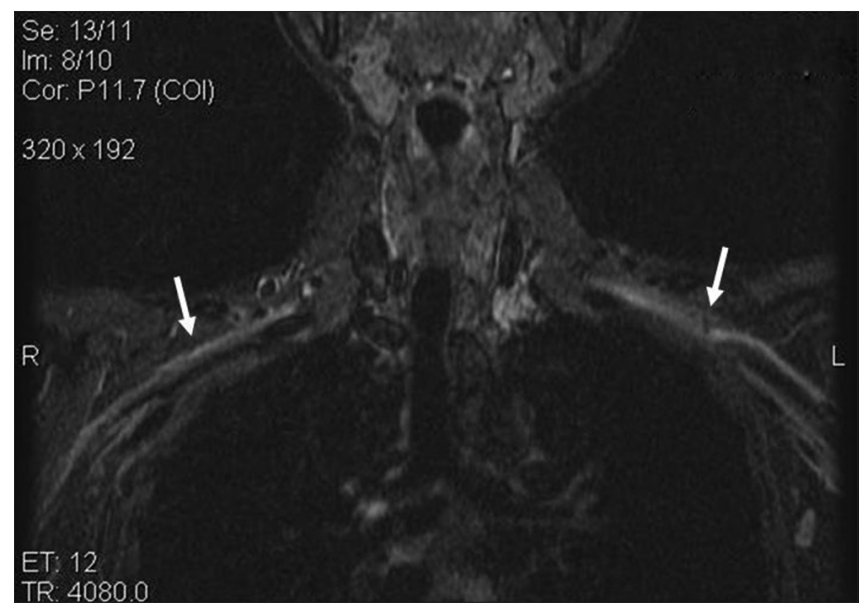

Figure 8: Coronal STIR images reveal hyperintense signal in divisions and cords of brachial plexi bilaterally in a patient in postpartum period (10 days after delivery) suggestive of brachial plexitis

Neuropraxic injury is seen as T2 hyperintense signal in the roots, trunks, or cords with or without enlargement [Figure $3 \mathrm{~A}$ and B]. Nerve ruptures are seen as discontinuity in the neural structures [Figure $4 \mathrm{~A}$ and $\mathrm{B}]{ }^{\left[{ }^{[1]}\right.}$ Associated findings of denervation edema in the muscles may be seen [Figure 5A and B].

Brachial plexus injuries may be associated with injuries to the subclavian artery due to their anatomical proximity to each other. Also post-traumatic pseudoaneurysm of subclavian artery may present with delayed brachial plexus paralysis due to compression of the brachial plexus ${ }^{[5]}$ [Figure 6].

\section{Nontraumatic Brachial Plexus Pathologies}

\section{Radiation fibrosis}

Patients undergoing radiation therapy in axillary region,

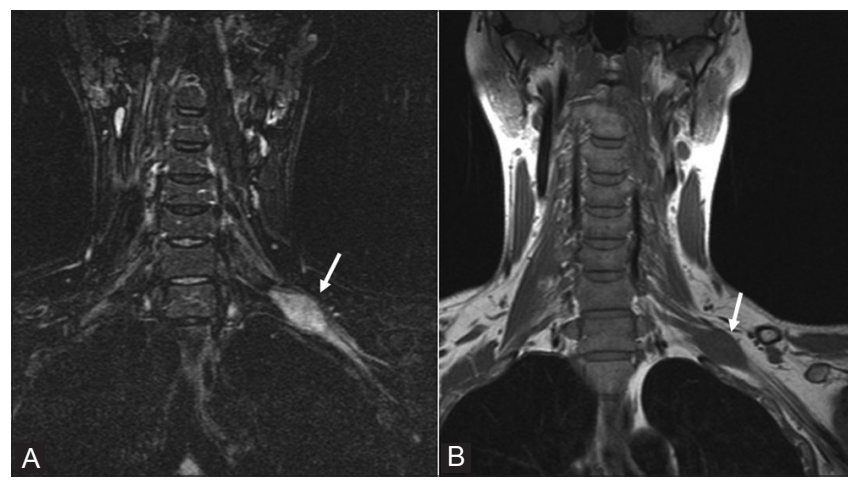

Figure 9 (A, B): Coronal STIR and T1-weighted images demonstrate a well-defined fusiform lesion involving left brachial plexus. It is isointense on T1-weighted images and hyperintense on STIR images suggestive of nerve sheath tumor

most commonly for breast carcinoma, may present with brachial plexopathy after several months to years. Radiation fibrosis is seen as diffuse thickening of the brachial plexus and iso- or hypointensity on T1- and T2-weighted images. The absence of a focal mass differentiates it from metastatic disease [Figure 7A-C]. Moreover, metastases appear hypointense on T1-weighted images and hyperintense on T2-weighted images. ${ }^{[6]}$

\section{Brachial plexus neuritis}

Acute brachial plexitis presents with severe shoulder and upper arm pain lasting for few days to weeks followed by upper arm weakness. Idiopathic brachial neuritis is of unknown cause but an immune-mediated inflammatory reaction following viral infection, vaccination, surgery, pregnancy, etc., has been proposed as etiology ${ }^{[7]}$ Bilateral brachial plexus neuritis in postpartum period [Figure 8] has been described by Dumitru and Liles. ${ }^{[8]}$ Brachial plexitis is seen on MRI as focal or diffuse hyperintense signal in brachial plexus. 


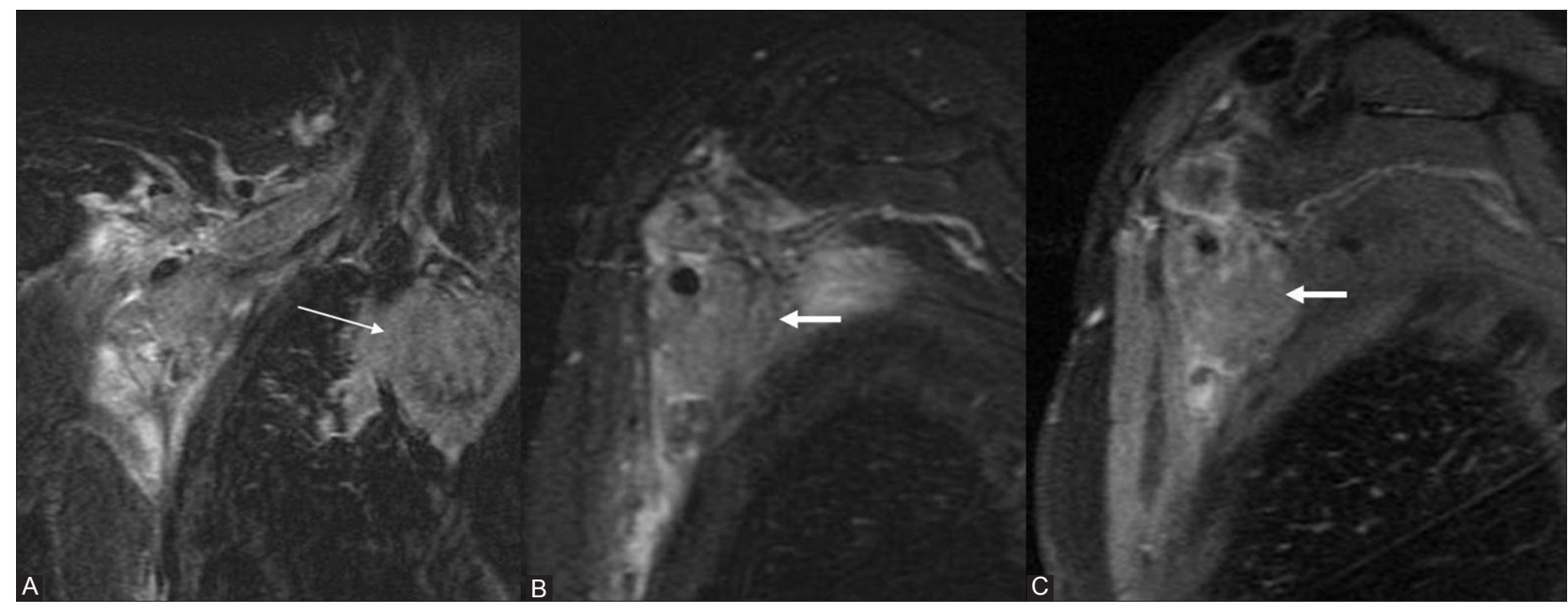

Figure 10 (A-C): Coronal and sagittal STIR and contrast-enhanced, fat-saturated, T1-weighted images reveal a well-defined mass encasing the cords of the right brachial plexus (thick short arrows) suggestive of metastases in a patient with breast carcinoma. The mass is hyperintense on STIR images and reveals mild almost homogeneous enhancement. Also seen is metastatic mediastinal lymphadenopathy (thin arrow)

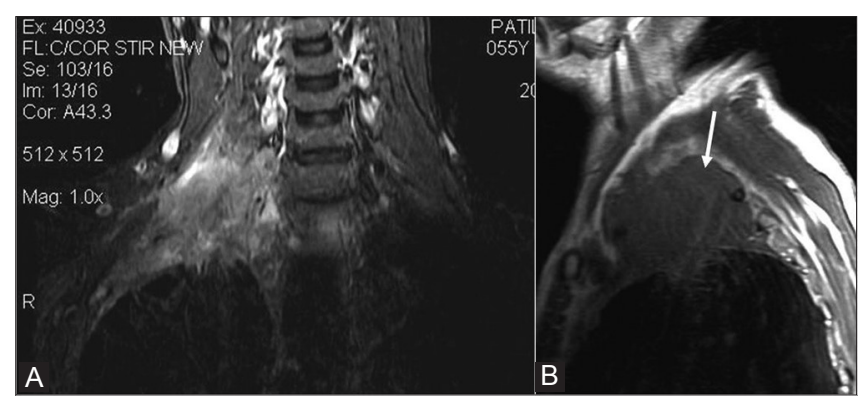

Figure 11 (A, B): Coronal STIR and sagittal T1-weighted images reveal right-sided superior sulcus (Pancoast) tumor seen as an ill-defined lesion involving roots and trunks of right brachial plexus

Brachial plexus tumors

Nerve sheath tumors (schwannoma and neurofibroma) are seen as ovoid lesions isointense to muscle on T1-weighted images and hyperintense on T2-weighted images with 'target' sign. These reveal intense enhancement on administration of gadolinium contrast.[Figure 9A and B]. Most common benign tumors that involve brachial plexus are lipomas and aggressive fibromatosis. ${ }^{[9,10]}$ Metastatic breast carcinoma [Figure 10A-C], superior sulcus tumors (non-small-cell lung carcinoma arising from lung apex) [Figure 11A and B], and lymphoma involve the brachial plexus frequently. ${ }^{[6]}$

\section{Thoracic outlet syndrome}

It is dynamically induced compression of neural and/or arterial structures crossing the cervicothoracobrachial junction. MRI plays an important role in demonstrating neurovascular compression, localizing it and identifying the structure causing the compression. The three spaces that are evaluated on sagittal T1-weighted images are the interscalene triangle, costoclavicular, and retropectoralis minor spaces with arm in neutral as well as hyperabducted position to look for compression

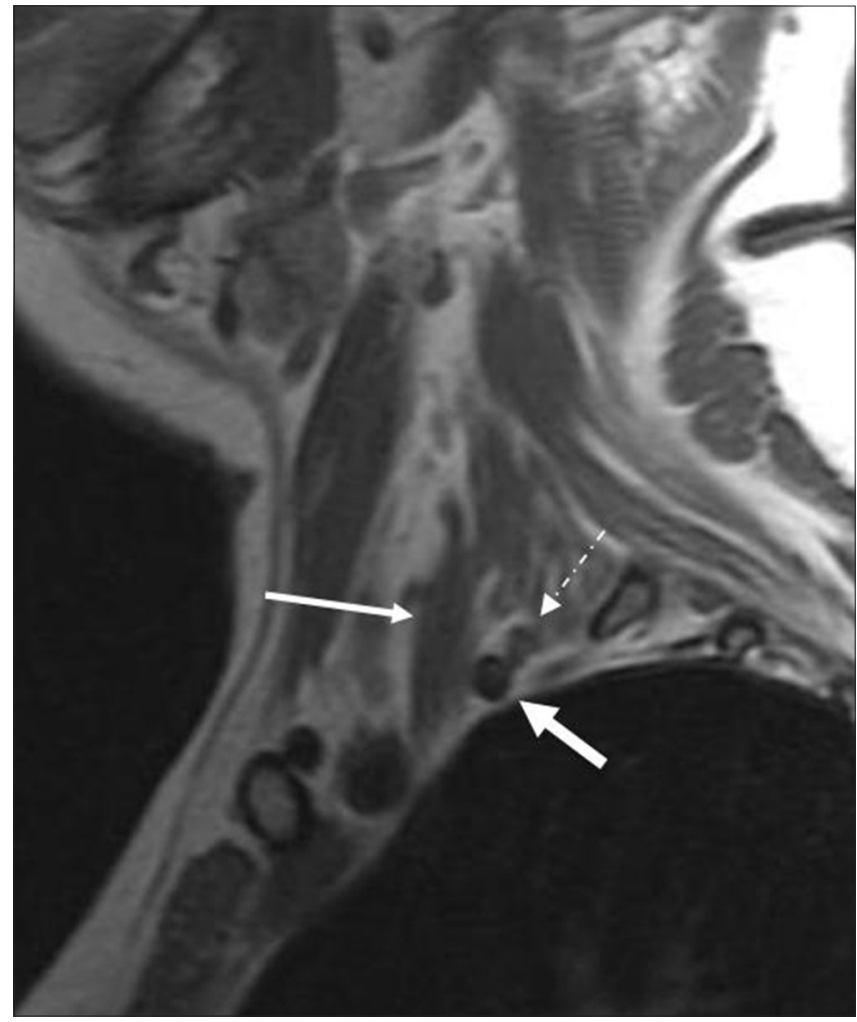

Figure 12: Sagittal T1-weighted image with arm in neutral position shows normal interscalene triangle. It is bounded anteriorly by scalenus anterior muscle (thin arrow). The subclavian artery (thick arrow) and roots of brachial plexus (arrow with dashed line) are seen

of the neurovascular structures [Figures 12-15]. The costoclavicular space is the most common site of compression followed by interscalene triangle. The lesions causing compression may be bony abnormalities (cervical rib [Figures 16 and 17], long transverse process of C7 vertebra, callus or osteochondroma of clavicle or first rib) or soft tissue pathologies (fibrous band, hypertrophy of scalenus anterior muscle, scalenus minimus muscle, 
and fibrous scarring). ${ }^{[11]}$ Bilateral hypoplastic first ribs fused to second ribs can also cause thoracic outlet syndrome [Figures 18 and 19]. Contrast-enhanced MR angiography may be performed with the arm in elevated position to demonstrate narrowing of subclavian artery. Dymarkowski, et al., recommend performing 3D MR angiography with arm in adduction followed by arm in hyperabducted position. ${ }^{[12]}$

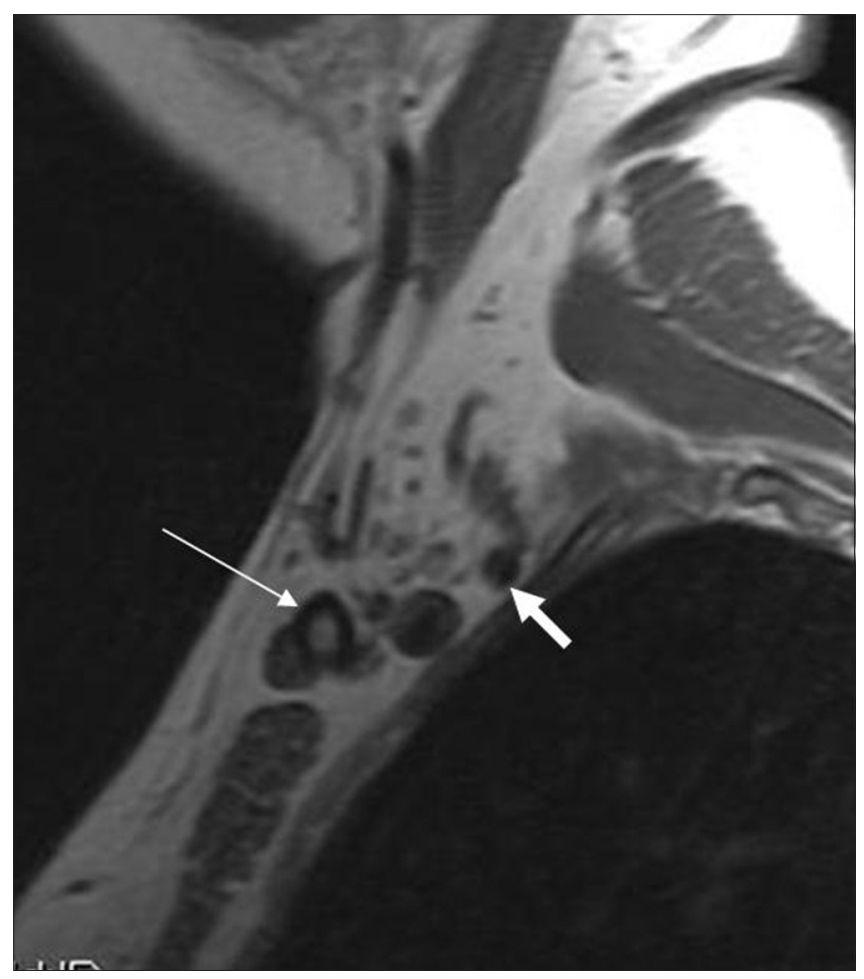

Figure 13: Sagittal T1-weighted image with arm in neutral position shows normal costoclavicular space between the clavicle and first rib. Thin arrow depicts clavicle while the thick arrow shows normal subclavian artery
To summarize, brachial plexus has a complex anatomy and long course. Various traumatic and nontraumatic pathologies affecting it can be evaluated optimally by MRI. Knowledge of the anatomy and proper planning of the scan are essential for complete evaluation of the brachial plexus.

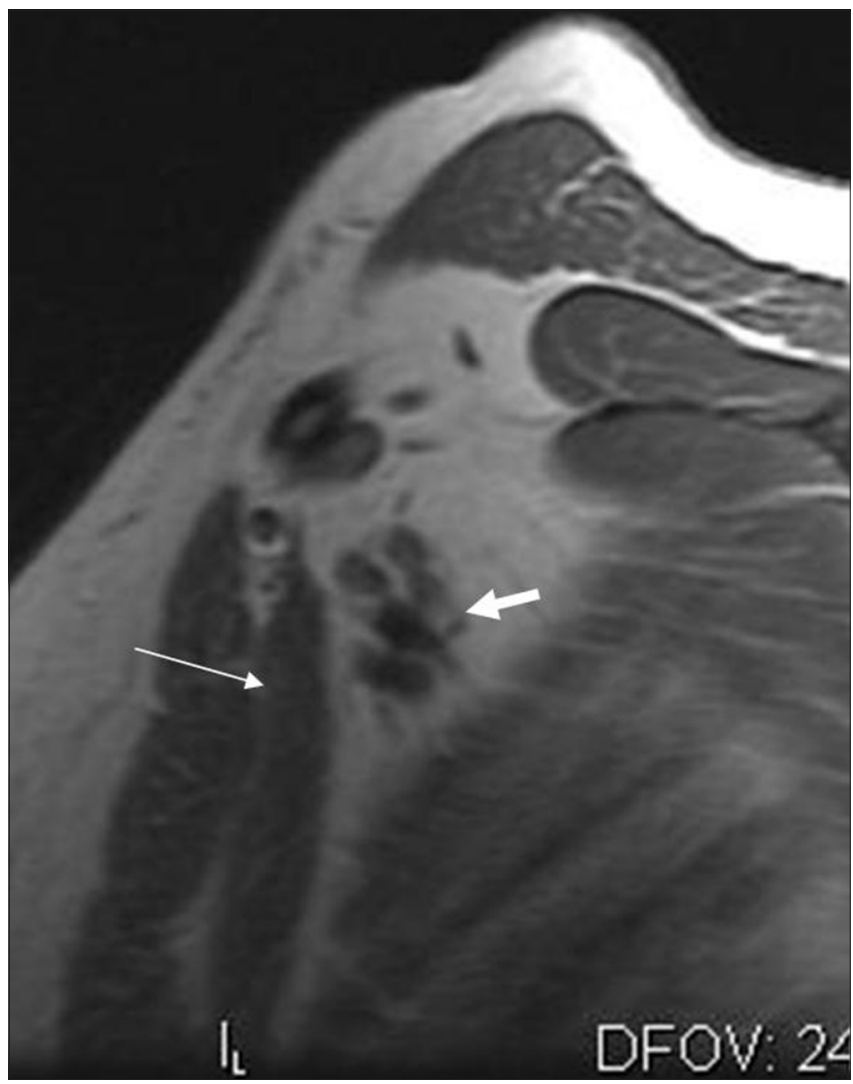

Figure 14: Sagittal T1-weighted image with arm in neutral position shows normal retropectoralis minor space. Thin arrow shows pectoralis minor muscle. The subclavian artery is depicted by thick arrow. The cords of brachial plexus lie superior to it

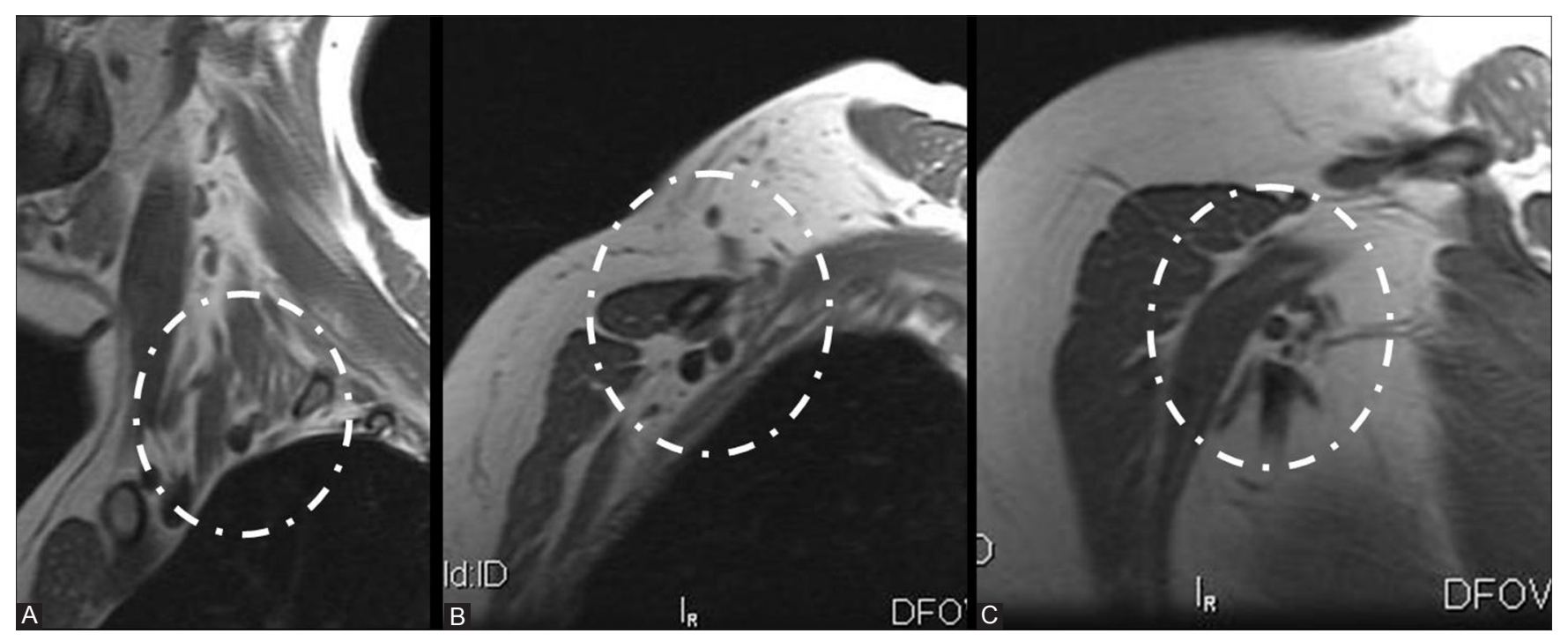

Figure 15 (A-C): Sagittal T1-weighted images with arm in hyperabduction demonstrating the interscalene triangle, costoclavicular, and retropectoralis minor spaces, respectively. Note decrease in the sizes of the latter two spaces during abduction 


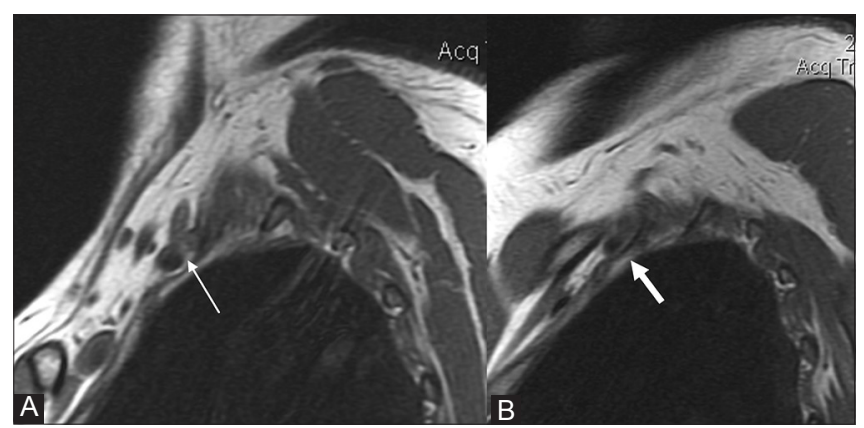

Figure 16 (A, B): Sagittal T1-weighted images with arm in neutral and hyperabducted positions reveals compression of subclavian artery and brachial plexus in costoclavicular space due to a cervical rib

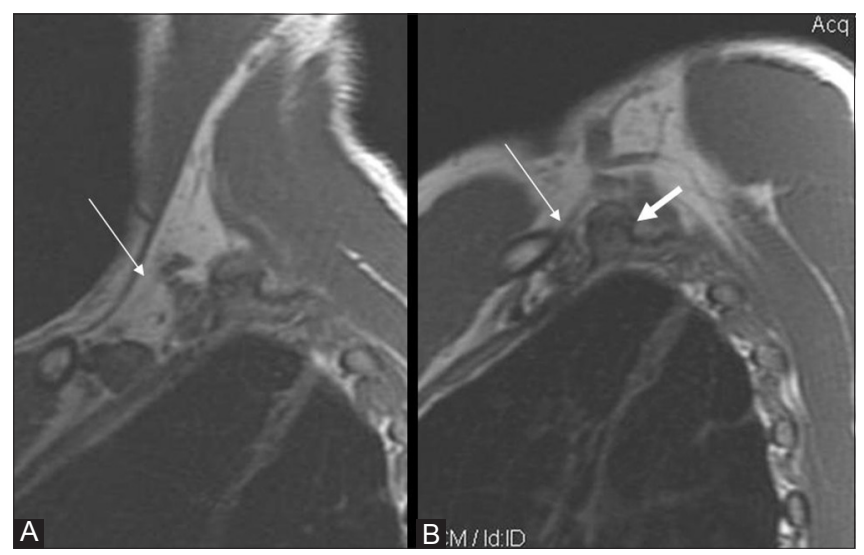

Figure 18 (A, B): Sagittal T1-weighted images with arm in neutral and hyperabducted positions show severe compression of subclavian artery and brachial plexus in costoclavicular space by an osseous structure

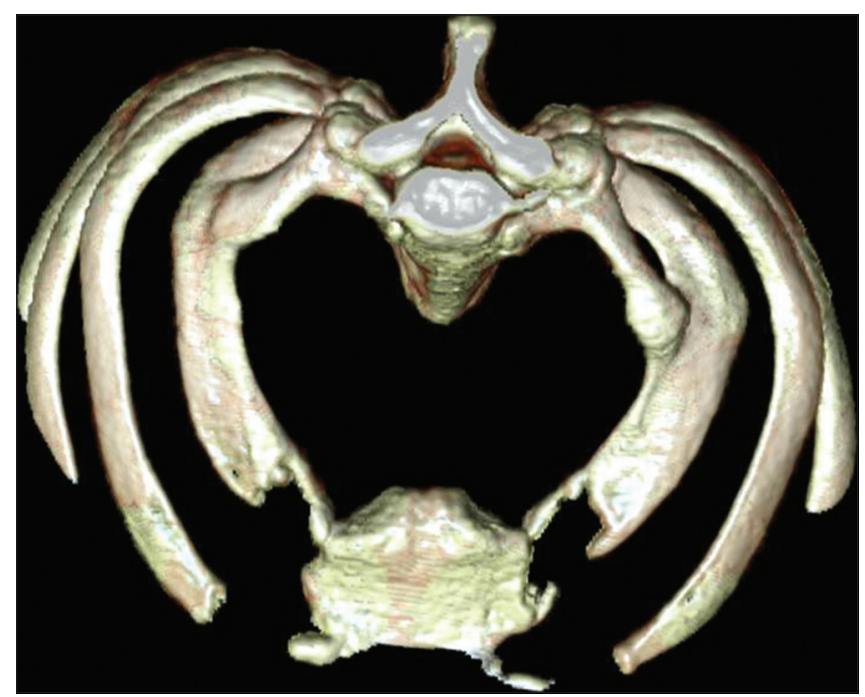

Figure 19: 3D CT of the same patient reveals bilateral hypoplastic first ribs fused to second ribs

\section{References}

1. Gerevini S, Mandelli C, Cadioli M, Scotti G. Diagnostic value and surgical implications of the magnetic resonance imaging in the management of adult patients with brachial plexus pathologies. Surg Radiol Anat 2008;30:91-101.

2. Bowen BC, Pattany PM, Saraf-Lavi E, Maravilla KR. The brachial plexus: Normal anatomy, pathology, and MR imaging. Neuroimaging Clin N Am 2004;14:59-85.

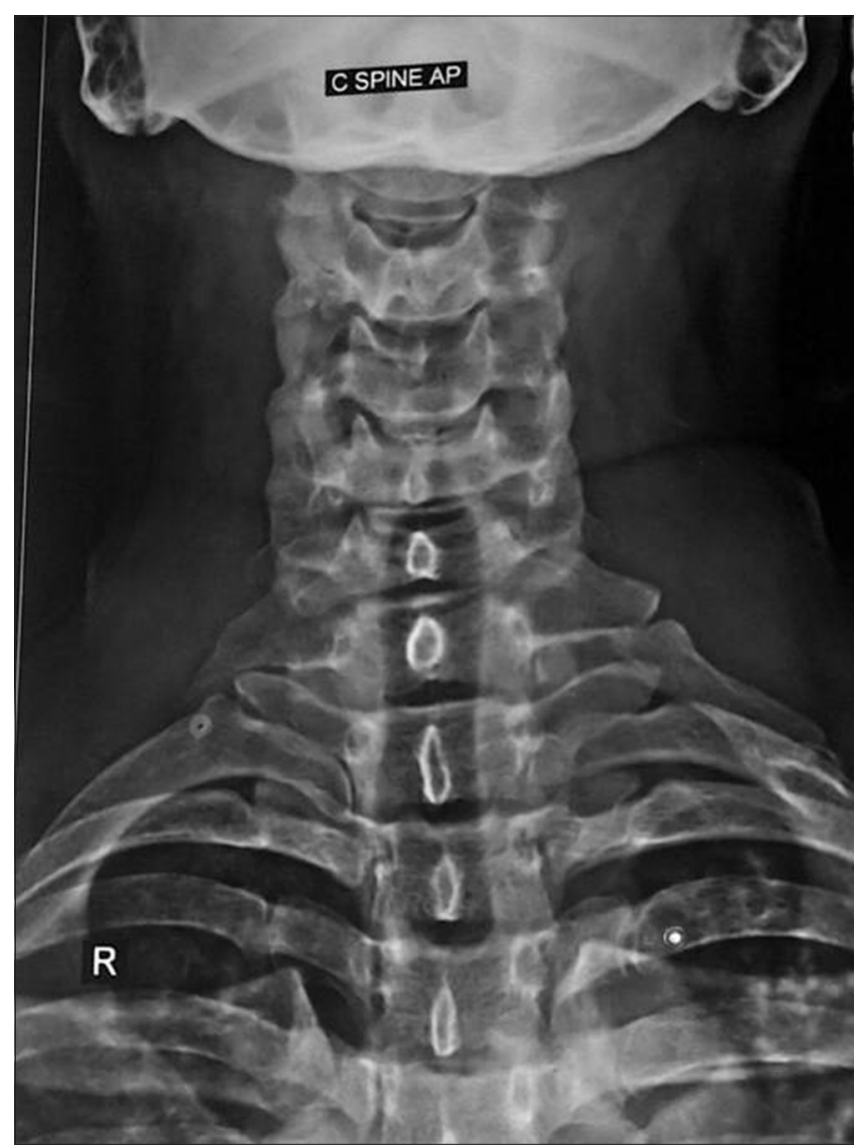

Figure 17: AP radiograph of cervical spine of this patient shows bilateral cervical ribs

3. Nagano A. Treatment of brachial plexus injury. J Orthop Sci 1998;3:71-80.

4. Yoshikawa T, Hayashi N, Yamamoto S, Tajiri Y, Yoshioka N, Masumoto T, et al. Brachial Plexus Injury: Clinical Manifestations, Conventional Imaging Findings, and the latest imaging techniques. RadioGraphics 2005;26:S133-43.

5. Hansky B, Murray E, Minami K, Körfer R. Delayed brachial plexus paralysis due to subclavian pseudoaneurysm after clavicular fracture. Eur J Cardiothorac Surg. 1993;7:497-8.

6. Wittenberg KH, Adkins MC. MR Imaging of Nontraumatic Brachial Plexopathies: Frequency and Spectrum of Findings. Radio Graphics 2000;20:1023-32.

7. Gonzalez-Alegre P, Recober A, Kelkar P. Idiopathic Brachial Neuritis. Iowa Orthop J 2002;22:81-5.

8. Dumitru D, Liles RA. Postpartum idiopathic brachial neuritis. Obstet Gynecol 1989;73:473-5.

9. Van Es HW, Bollen TL, van Heesewijk HP. MRI of the brachial plexus: A pictorial review. Eur J Radiol 2010;74:391-402.

10. Saifuddin A. Imaging tumours of the brachial plexus. Skeletal Radiol 2003;32:375-87.

11. Demondion X, Herbinet P, Van Sint Jan S, Boutry N, Chantelot C, Cotten A. Imaging assessment of thoracic outlet syndrome. RadioGraphics 2006;26:1735-50.

12. Dymarkowski S, Bosmans H, Marchal G, Bogaert J. Three-dimensional MR angiography in the evaluation of thoracic outlet syndrome. AJR Am J Roentgenol 1999;173:1005-8. 1

Cite this article as: Lawande M, Patkar DP, Pungavkar S. Pictorial essay: Role of magnetic resonance imaging in evaluation of brachial plexus pathologies. Indian J Radiol Imaging 2012;22:344-9.

Source of Support: Nil, Conflict of Interest: None declared. 